\title{
EFFECTIVE BANDWIDTH ANALYSIS OF MIMO BASEd Mobile Cloud Computing
}

\author{
${ }^{1}$ Suremya Varghese and ${ }^{2}$ Ganesan Subramanian \\ ${ }^{1}$ PG Student, School of Engineering \& IT, Manipal University, Dubai \\ ${ }^{2}$ Assistant Professor, School of Engineering \& IT, Manipal University, Dubai
}

\begin{abstract}
Digital Disruption is all around us. Mobile is overtaking desktop, Social Media is beating search, Messaging Application are challenging e-mails and everything around us is becoming connected. Mobile devices especially the smart phones are fueling the culture of "Anytime, Anywhere, And Anything". Smartphone is not only ubiquitous but also the primary computing device for many.These paradigm shifts are fueled by the explosive growth of smart phones which has touched a volume of 1.6 billion units globally. Smartphone growth has also triggered the explosive growth of mobile applications and cloud computing .Together, Mobile cloud computing is now a potential technology for mobile services .MCC overcomes obstacles related to battery life, storage capacity and low bandwidth. Current smart phones uses $2 \times 2$ MIMO which gives a speed 300Mbps, by using massive MIMO technology speed can be enhanced up to 1Gbps. This paper gives a BER (Bit Error Ratio) analysis to prove that by increasing number of transmitting and receiving antennas the performance can be enhanced.
\end{abstract}

\section{KEYWORDS}

BER, Cloud computing, mobile, MCC, MIMO

\section{INTRODUCTION}

Mobile devices and apps are becoming an essential part of human life. Mobile devices are most convenient way of communication which are not bound by time and place. Mobile users enjoy various services from mobile applications, which run on the device or/and on remote servers via wireless networks. However, mobility is facing many problems such as resource scarceness, finite energy and low connectivity. Mobile cloud computing can address these problems by executing mobile applications on resource providers external to the mobile device, on the cloud.

\subsection{Cloud Computing}

"Cloud computing refers to both the applications delivered as services over the internet and the hardware and systems software in the data centres that provide those services"[3].In the simplest terms, services and solutions that are delivered and consumed in real time over internet are cloud services. For example when you store your photos online or using a social media site or e-mails, you are using a "cloud computing service". Just as a "cloud" in the sky is diffuse and capable of hiding things, a "cloud network" is a diffuse network of computers connected in a hidden fashion [2]. Cloud computing allows users to use infrastructure (server, network and storage), platforms (middleware services and OS) and software's (application programs). 
Clouds can be Public clouds - providers shares resources over internet to public. Private clouds dedicated and secured clouds or Hybrid clouds - and integration of public and private clouds.

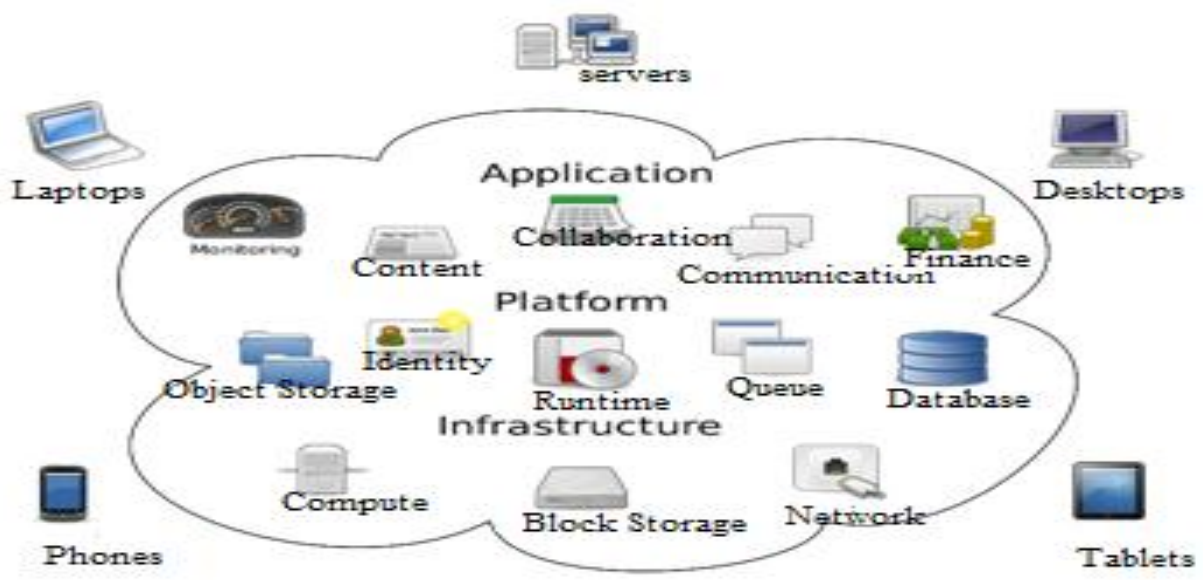

Figure 1 Cloud Architecture.

\subsection{Mobile Cloud Computing}

Themobile cloud computing is a development of mobile computing, and an extension to cloud computing. In mobile cloud computing, mobile device based intensive computing, data storage, mass information processing have been transferred to cloud, which are then accessed over the wireless connection based on a thin client. This reduces the computing capability and storage requirement of mobile devices. This brings mobile cloud computing not just smart phone users but also to simple mobile phone users. Mobile clouds are changing the work culture from brick and mortar office to just an internet connected phone. 


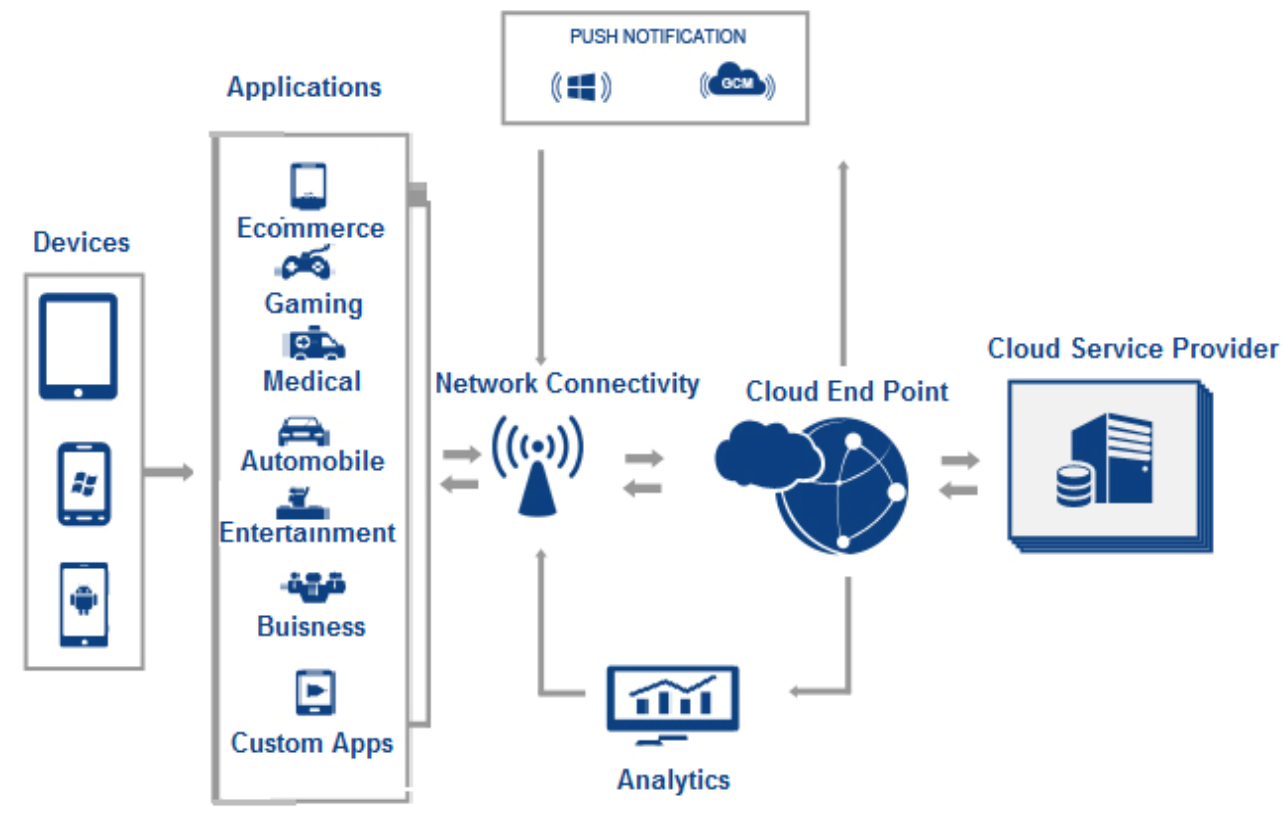

Figure 2---MCC architecture.

\subsubsection{Advantages:}

1. Improved battery life - As the dependency on mobile devices are increasing the battery life become critical. By offloading the power consuming computations to the cloud, computation time and power can be saved. For example, offloading a compiler optimization for image processing can reduce $41 \%$ for energy consumption of a mobile device [1].

2. High data storage capacity - Users can store and access large data on cloud using wireless which overcomes the limited storage of mobile devices.

3. Increases reliability and availability - By storing data and applications on cloud reduces the chances of data lost from device. And with an internet connection users can access it from anywhere.

4. Improved processing power - By sharing computational work with resourceful cloud improves processing time and power.

\subsubsection{ISSUES}

The mobile cloud computing services is still in early stages of development and facing several issues and challenges like,

1. Security and privacy--Protecting user privacy and data/application is of concern. Mobile devices are exposed to numerous security threats like malicious codes, hacking and viruses. Some GPS services gives out information about user's location [20].

2. Computing issues - Even though computational offloading is one the main features of MCC it faces some issues like selection of data/application to be offloaded and 
International Journal on Cybernetics \& Informatics (IJCI) Vol. 5, No. 2, April 2016

dynamically updating of that files. Sometimes it may not save energy and time as expected [19].

3. Network connectivity-- since MCC is based on internet, the quality of network connectivity is very important. But it faces issues like longer latency of wireless connections, frequent disconnections, traffic congestion, weather conditions and mobility of users.

Due to limited radio sources the bandwidth is low and the high speed internet is not available everywhere. There should be a seamless connection handover between different access schemes (3G, 4G, GPRS, and WLAN Etc.).

4. Mobile application issues: Mobiles devices uses different operating system the cloud should be able to handle data across multiple O.S. To get the advantage of cloud computing data should be distributed properly.

\subsection{MIMO (MULTIPLE INPUT MULTIPLE OUTPUT)}

Compared to normal wired channels, the wireless communication is suffered by the multipath radio channel between the transmitter and receiver and the scarcity of available spectrum. Antenna diversity can take advantage of multiple signals to improve the transmission. The various possible configurations are shown in Figure 1.3, are referred as Single Input Single Output (SISO), Single Input Multiple Output (SIMO), Multiple Input Single Output (MISO) and Multiple Input Multiple Output(MIMO). SIMO is a form of receive diversity and MISO uses transmit diversity. MIMO combines transmit and receive diversity.
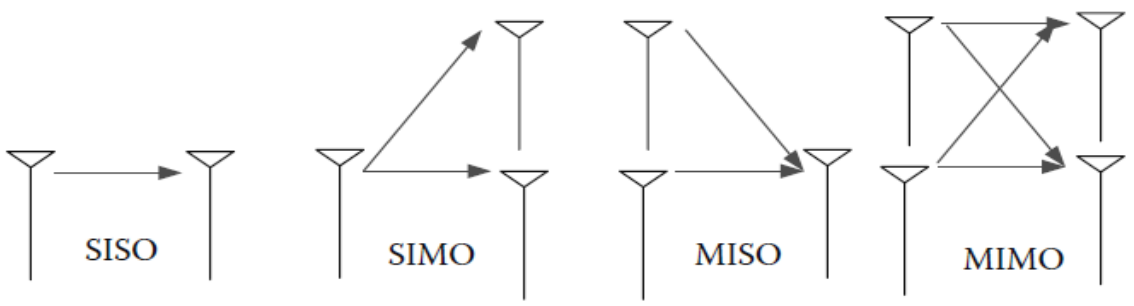

Figure 3-Multiple Antenna Configurations.

Each antenna element on a MIMO system operates on the same frequency and therefore does not require extra bandwidth. A MIMO system consumes no extra power due to its multiple antennas as the total power through all antenna is less than or equal to that of a single antenna system [4].

In current $3 \mathrm{~g}$ and $4 \mathrm{~g}$ communications traditional MIMO $(2 \times 2)$ is used. When we increases the number of antennas the capacity of the system increases linearly. But after 8 antennas, due to size and position issues of antennas the capacity do not increase. Latest studies show that if number of antennas is increased in the range of 100 the capacity improved well -known as massive MIMO [5]. 


\section{RELATED WORK}

Advances in computing and communication has made Mobile cloud computing an attractive area of research over the past few years. There have been a considerable number of researches to provide MCC with better coverage and quality, power and bandwidth efficient in all diverse environment.

Article [10] describes how computers configured to use in single location changed to portable devices with mobile computing and wireless connection. It also highlights the challenges of mobile computing like low bandwidth, address migration, low power and risk to data.

Article [8] explains the need for mobile cloud computing, advantages of MCC, current approaches, its issues and suggested some improvement methods. Some researchers [11],[7],[14] describes the architecture, applications and challenges of mobile cloud computing.

Yi $\mathrm{Xu}$ And Shiwen Mao reviewed mobile cloud computing, with focus on the technical challenges of MCC for multimedia applications [9].

The article [5] presents an overview of potential network architecture and some of the potential technologies to employed in future $5^{\text {th }}$ Generation Systems which includes

Non orthogonal multiple access (NOMA), Full duplex, Device to Device communication, Cognitive radio, millimeter Wave communication and Massive MIMO.

With the help of a simple 2 branch transmit diversity scheme, article [6] explains antenna diversity is most practical and effective technique to reduce multipath fading.

In [4], the author provided an overview of MIMO systems and MIMO channel modelling techniques. With the help of MATLAB simulations showed that equal diversity gain does not imply equal performance, but limited their analysis till 4X4 MIMO.

Some researchers analysed performance of 4X4 MIMO- OFDM hybrid technology using different modulation schemes like QPSK and 16 QAM [16], [17]. In [18], the performance of 4X4 MIMO is analysed practically in laboratory.

\section{RESEARCH OBJECTIVES}

This objective of this research is to identify and present recommendation for improving transmission efficiency in Mobile Cloud Computing. This is done analysing the BER performance mathematically and simulating in MATLAB SIMULINK. This research is done in three phases

Phase 1: Analysis the BER performance of different modulation techniques

Phase 2: Analysis the BER performance of different fading channels.

Phase 3: Analysis the BER performance multiple antenna configurations. 


\section{ANALYSIS}

BER (Bit Error Ratio) testing is a powerful methodology for testing digital transmission system. In simple terms BER is the ratio of number of total errors tothe total number of transferred bits during the time interval. BER is a unit less performance measure, often expressed as a percentage. BER depend on transmitter, receiver and the medium between them.

\subsection{BER ANALysis OF DifFerent MOdulation TECHNiQUES}

The most fundamental digital modulation techniques are:

- $\quad$ PSK (phase-shift keying)

- FSK (frequency-shift keying)

- $\quad$ ASK (amplitude-shift keying)

- QAM (quadrature amplitude modulation).

Simulation have been done using MATLAB, for BPSK, BDPSK, BFSK and 16 QAM. For the purpose of simulation different SNR (Eb/No) is introduced (-5db to $15 \mathrm{db}$ ) in the AWGN channel. Figure 4 shows the performance of different modulation in AWGN CHANNEL. In the graph as $\mathrm{Eb} / \mathrm{No}$ increases the BER decreases for all modulation.

Graph and the table values shows that BPSK has better performance. QPSK performance is very similar to BPSK. All carrier signals other than BPSK and QPSK have some level of correlation between their signals which make it to harder to identify the signal at receiver end. This makes BPSK and QPSK to have lowest bit error.

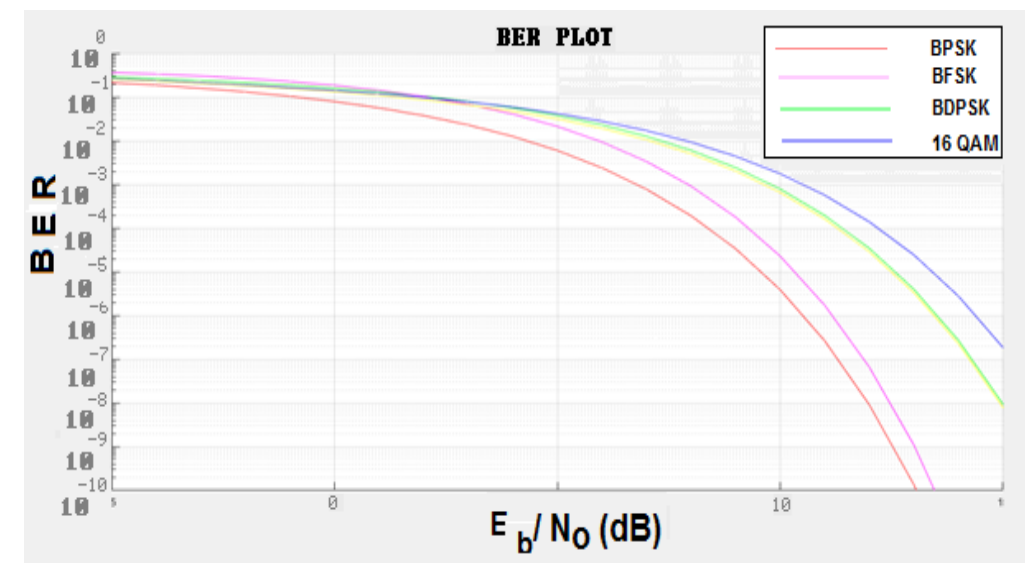

Figure 4. BER Analysis for Different modulation 
International Journal on Cybernetics \& Informatics (IJCI) Vol. 5, No. 2, April 2016

Table 1. Theoretical BER Calculation

\begin{tabular}{|c|c|c|}
\hline $\begin{array}{c}\text { MODULATION } \\
\text { TECHNIQUE }\end{array}$ & FORMULE & $\begin{array}{c}\text { BER WHEN } \\
\left(E_{b} \mid N_{o}\right)=5\end{array}$ \\
\hline BPSK & $P_{b}=Q\left(\sqrt{\frac{2 E_{b}}{N_{o}}}\right)$ & 0.0008 \\
\hline BFSK & $P_{b}=Q\left(\sqrt{\frac{E_{b}}{N_{o}}}\right)$ & 0.0129 \\
\hline BDPSK & $P_{b}=\frac{1}{2} \exp \left(-\frac{E_{b}}{N_{o}}\right)$ & 0.0036 \\
\hline 16 QAM & $P_{b} \cong \frac{3}{4} Q\left(\sqrt{\frac{4 E_{b}}{5 N_{o}}}\right)$ & 0.0171 \\
\hline
\end{tabular}

\subsection{BER ANALYSIS OF DifFERENT ChaNNELS.}

The above simulation is done considering only AWGN channel but signals undergo fading due to multipath propagation, attenuation and scattering. Fading severally affects the performance of the system. It can be clearly understand from the graph, the BER is increased considerably in Rician and Rayleigh fading channels than AWGN channel. A Simulink model is used to estimate BPSK BER performance in AWGN, Rayleigh and Rician channel is shown in the graph with Doppler shift $=0.01 \mathrm{~Hz}$ jakes model.

Table 2. Theoretical BER Calculation

\begin{tabular}{|c|c|c|}
\hline CHANNEL & FORMULE & BER WHEN $\left(E_{b} \mid N_{o}\right)=5$ \\
\hline AWGN & $P_{b}=Q\left(\sqrt{\frac{2 E_{b}}{N_{o}}}\right)$ & 0.0008 \\
\hline RAYLEIGH & $P_{b}=\frac{1}{2}\left\lfloor 1-\sqrt{\frac{\overline{\gamma_{b}}}{1+\overline{\gamma_{b}}}}\right\rfloor$ & 0.0436 \\
\hline RICIAN $(\mathrm{k}=2)$ & $P_{b}=\frac{1}{2} \operatorname{erf} c\left\lfloor\sqrt{\frac{k \overline{\gamma_{b}}}{k+\overline{\gamma_{b}}}}\right\rfloor$ & 0.0455 \\
\hline
\end{tabular}




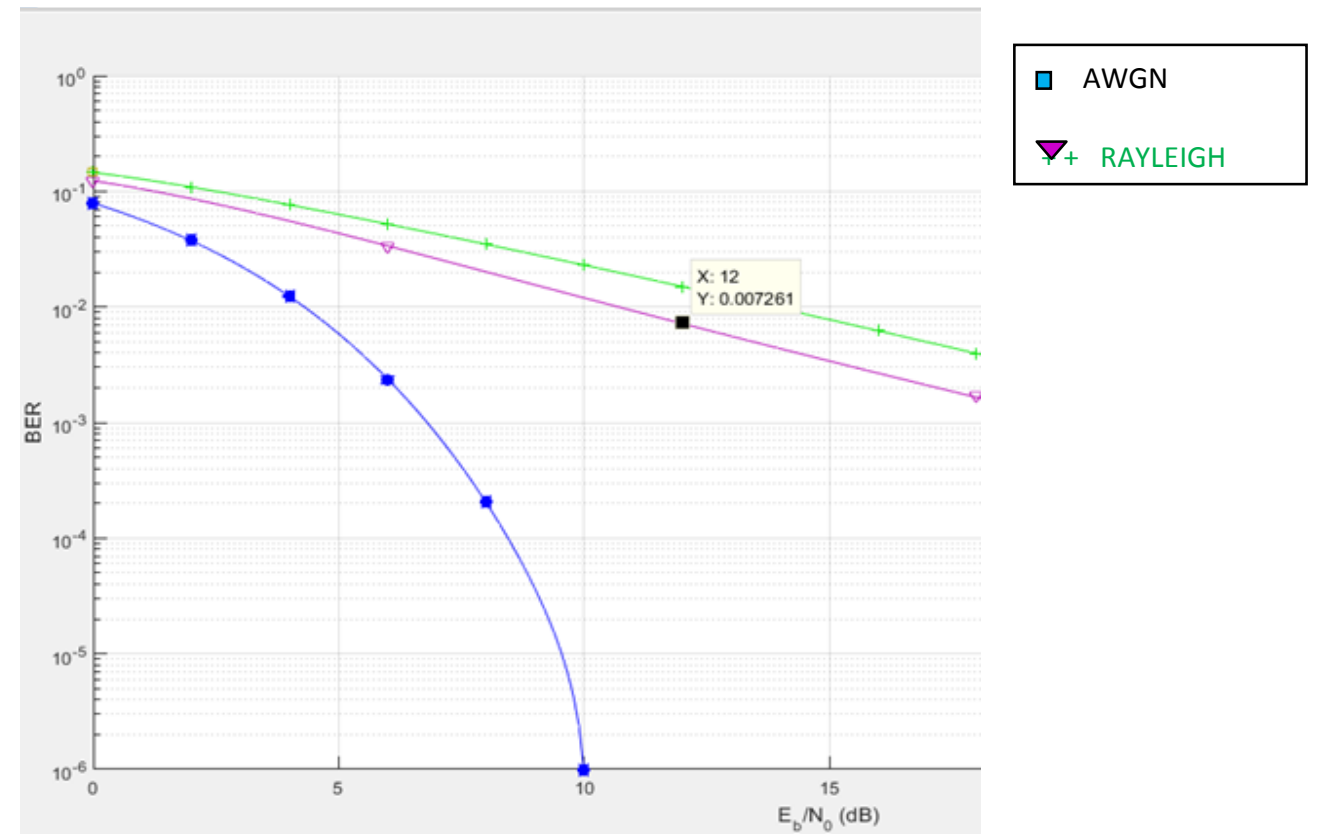

Figure 5 BER Analysis for fading channel

\subsection{MULTIPLE ANTENNA CONFIGURATIONS}

To order improve the data rate and reliability of communication over multipath fading channel, spatial diversity is used. Data is encoded by channel coding method Space Time Block Codes (STBC). The encoded data is split into $\mathrm{N}_{\mathrm{t}}$ streams that are simultaneously transmitted by $\mathrm{Nt}$ antenna. The received signal at each receiver linear super position of the $\mathrm{N}_{t}$ transmitted signal plus noise. Performance is determined by the matrices constructed from pairs of distinct set code sequences. The channel matrix can be written as

The received signal at antenna $\mathrm{j}$,

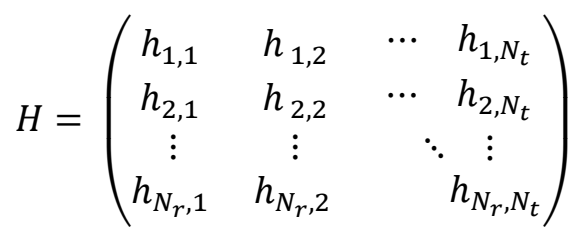

The received signal vector

$$
r_{t}^{j}=\sum_{i=1}^{N_{t}} h_{j, i} x_{t}^{i}+n_{t}^{j}
$$

$$
\begin{gathered}
r_{t}=\left(r_{t}^{1}, r_{t}^{2}, \ldots, r_{t}^{N_{r}}\right) \\
r_{t}=H_{t} x_{t}+n_{t}
\end{gathered}
$$

MIMO system with $\mathrm{N}_{\mathrm{t}}$ transmit antenna and $\mathrm{N}_{\mathrm{r}}$ receiving antenna has diversity gain equal to $\mathrm{N}_{\mathrm{t}} \mathrm{N}_{\mathrm{r}}$. Simulations are done with BPSK modulation in Rayleigh channel. 
International Journal on Cybernetics \& Informatics (IJCI) Vol. 5, No. 2, April 2016

\subsubsection{SIMO- SINGLE INPUT MULTIPLE OUTPUT}

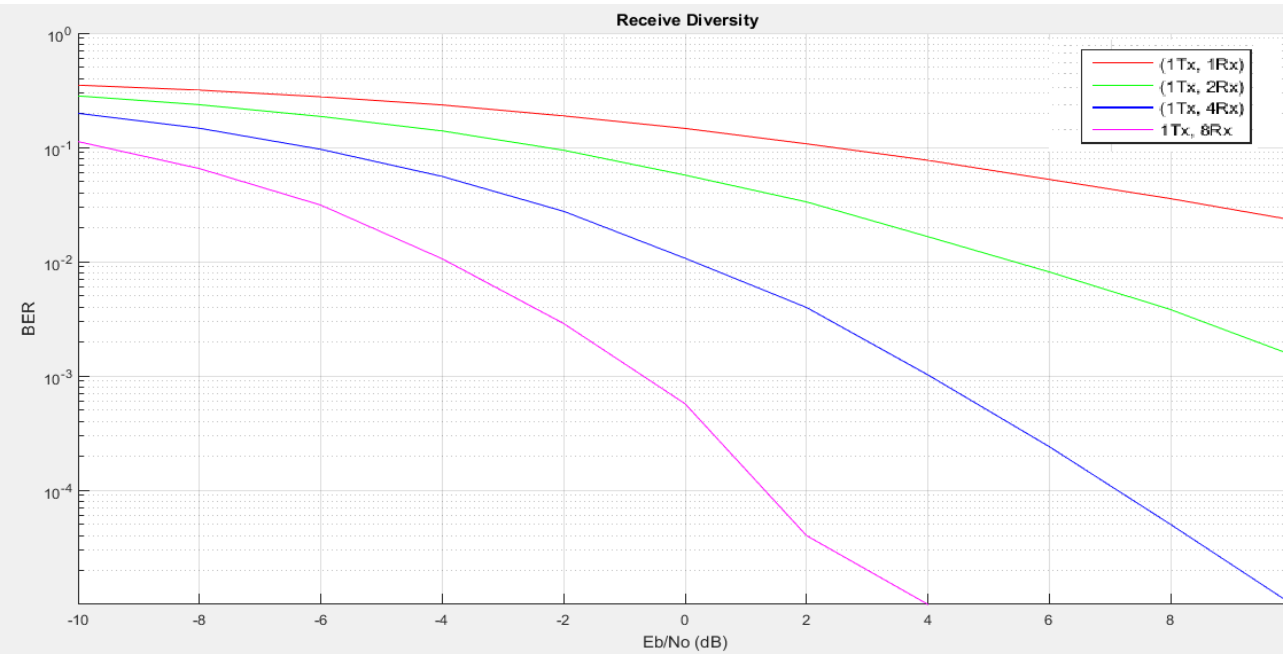

Figure 6.Receiver Diversity

Table 3. BER comparison of SIMO

\begin{tabular}{|c|c|c|c|c|}
\hline $\mathrm{Eb} / \mathrm{No}$ & $1 \times 1$ & $1 \times 2$ & $1 \mathrm{X} 4$ & $1 \times 8$ \\
\hline 0 & 0.1483 & 0.0578 & 0.03097 & 0.00032 \\
\hline 2 & 0.1082 & 0.0332 & 0.0033 & $06 \mathrm{E}-05$ \\
\hline 5 & 0.02415 & 0.00152 & $1 \mathrm{E}-05$ & 0 \\
\hline
\end{tabular}

\subsubsection{MISO- MULTIPLE INPUT SINGLE OUTPUT}

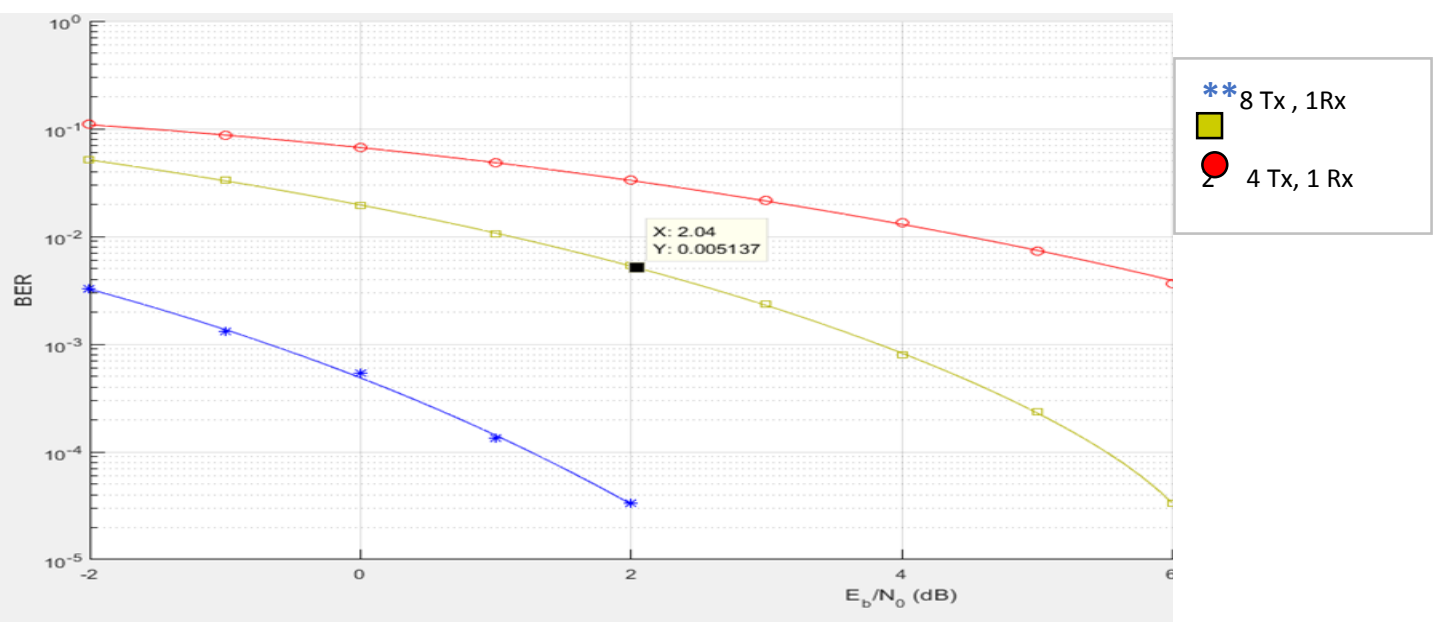

Figure 7. Transmitter Diversity 
International Journal on Cybernetics \& Informatics (IJCI) Vol. 5, No. 2, April 2016

Table 4. BER comparison of MISO

\begin{tabular}{|c|c|c|c|}
\hline $\mathrm{Eb} / \mathrm{No}$ & $2 \times 1$ & $4 \times 1$ & $8 \times 1$ \\
\hline 0 & 0.0671 & 0.033 & 0.0032 \\
\hline 2 & 0.0310 & 0.00513 & $5.3 \mathrm{E}-4$ \\
\hline 5 & 0.0042 & $9.2 \mathrm{E}-05$ & $1.3 \mathrm{E}-05$ \\
\hline
\end{tabular}

\subsubsection{MIMO- MULTIPLE INPUT MULTIPLE OUTPUT}

Table 5. BER comparison of MIMO

\begin{tabular}{|c|c|c|c|}
\hline $\mathrm{Eb} / \mathrm{No}$ & $2 \times 2$ & $4 \times 4$ & $8 \times 8$ \\
\hline 0 & 0.0380 & 0.002 & 0.001 \\
\hline 2 & 0.0152 & $4.33 \mathrm{E}-04$ & $3.3 \mathrm{E}-05$ \\
\hline 3 & 0.0082 & $1.33 \mathrm{E}-04$ & 0 \\
\hline
\end{tabular}

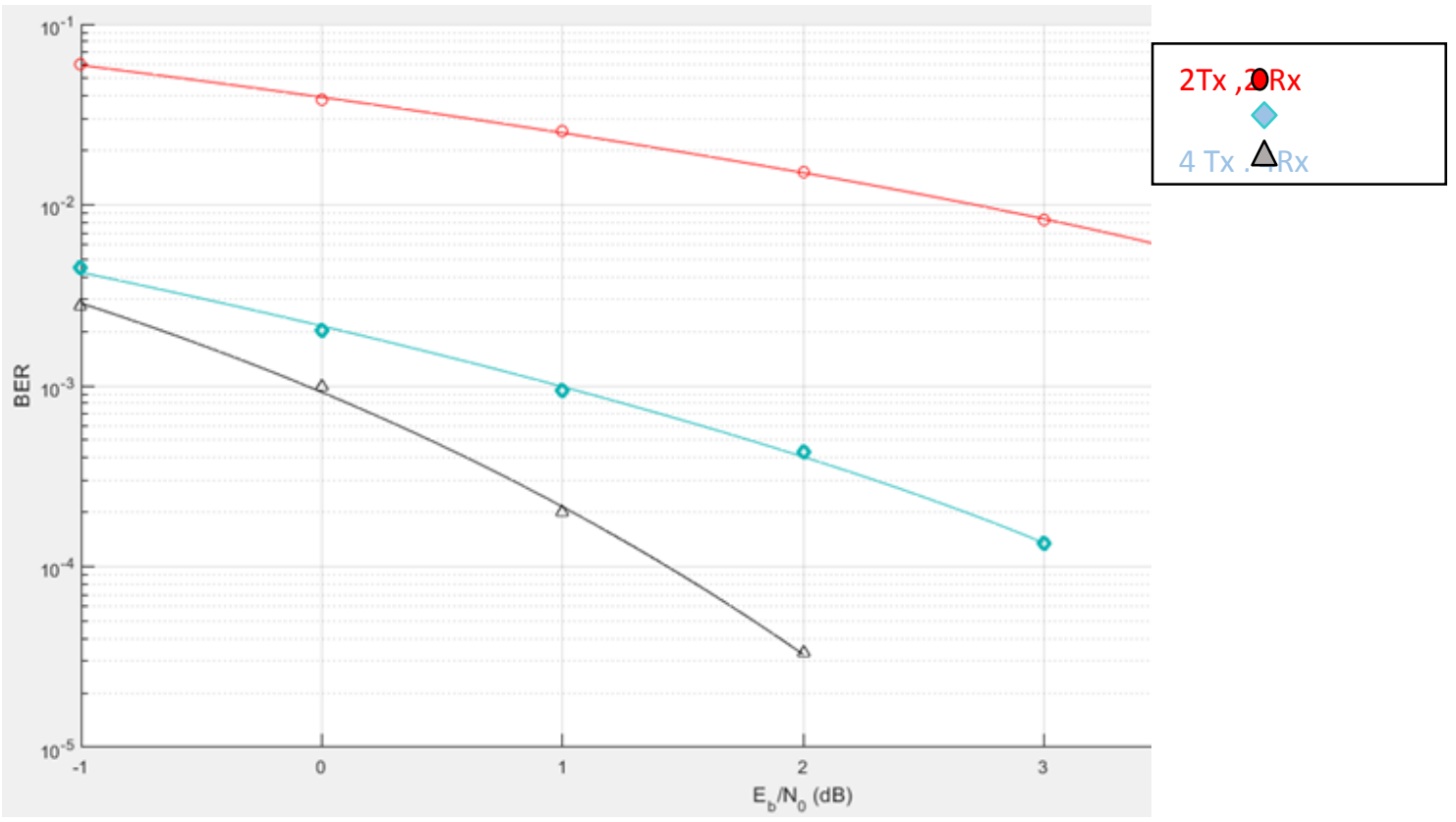

Figure 8. BER Analysis of MIMO 
International Journal on Cybernetics \& Informatics (IJCI) Vol. 5, No. 2, April 2016

\section{CONCLUSION}

This paper provided basic overview of mobile cloud computing and its need and challenges. We briefly discussed Space Time Block Coding with different antenna diversity. Its proved that as number of antenna increases the bit error rate also decreases. Eventhough diversity is same for SIMO 1X4, MISO 4X1 and MIMO 2X2 the BER is different. MIMO system will have higher capacity. Having more number of transmitter end is more feasble than at the receiver end as its easy to add atennas at base station depending upon requirment rather adding at each handset.We can propose MIMO 8X8 is better pereference for 5G integrated MCC.

\section{REFERENCES}

[1] S.C Hsueh, J.Y Lin, M.Y Lin, "Secure cloud storage for conventional data archive of smart phones," in: Proc. $15^{\text {th }}$ IEEE Int. Symposium on Consumer Electronics, ISCE '11, Singapore, June 2011.

[2] B.Gabriel, "NASA Turns to Online Giant Amzone for Cloud Computing Services for Mars Rover Curiosity," 12 August 2012 . [Online]

[3] Miss Priyanka J. Pursani, Prof. P.L Ramteke, "Mobile Cloud Computing," : International Journal of Advanced Research in Computer Engineering And Technology(IJARCET), Volume 2, Issue 4, April 2013.

[4] Luis Miguel Cortes-Pena, "MIMO Space time block coding (STBC): Simulation and Results", Design Project, Personal and Mobile Communications, Georgia Tech, April-2009.

[5] M.A Zheng, ZHENG Zheng Quan, DING ZhiGuo, FAN PingZhi and LI HengCHao," Key Techniques for 5G wireless communications, Network Architecture, physical layer and MAC layer Perspective", Science China, Vol 58, April 2015.

[6] S. M. Alamouti, , "A simple transmitter diversity scheme for wireless communication", IEEE Journal of Select. Areas Communications., vol. 16 ,Number 8, pp.1451 -1458, 1998.

[7] Hoang T. Dinh, Chonho Lee, Dusit Niyato*, Ping Wang, A Survey Of Mobile Cloud Computing: Architecture, Applications, And Approaches, Wireless Communications and Mobile Computing, Volume 13, Issue 18, December 2013, pp:1587-1611.

[8] Niroshinie Fernando', Seng W. Loke', Wenny Rahayu, Mobile cloud computing: A survey,Future Generation Computer Systems, Volume 29, Issue 1, January 2013, Pages 84-106.

[9] Yi Xu , Shiwen Mao, A Survey Of Mobile Cloud Computing For Rich Media Applications, IEEE Wireless Communications, June 2013, pp 46-53.

[10] George H. Forman and John Zahorjan, "The challenges of Mobile Computing", University of Washington. April 1994.

[11] Han Qi and Abdullah Gani, "Research on mobile cloud computing: Review, Trend and Perspectives", University of Malaya, Malaysia.

[12] J. V. Vishniakova, A. I. Luchaninov "application of antenna theory with nonlinear elements for mimo analysis" International

Conference on Antenna Theory and Techniques, 2013, Odessa, Ukraine

[13] Swati Chowdhuri, Sayan Chakraborty, Nilanjan Dey, Ahmad Taher Azar,. Mohammed AbdelMegeed M. Salem,, Sheli Sinha Chaudhury, Pranab Banerjee, Recent Research on Multi Input Multi Output (MIMO) based Mobile ad hoc Network: A Review, International Journal of Service Science, Management, Engineering, and Tech., 5(3), 54-65, July-Sept. 2014.

[14] Dipayan Dev and Krishna Lal Baishnab , "A Review and Research towards Mobile Cloud Computing" , 2014 2nd IEEE International Conference on Mobile Cloud Computing, Services, and Engineering,2014.

[15] Bernard Sklar, Digital Communications: Fundamentals andApplications - Prentice Hall Communications Engineering and Emerging Technology, Jan 2001. 
International Journal on Cybernetics \& Informatics (IJCI) Vol. 5, No. 2, April 2016

[16] Deepak Sharma , Praveen Srivastava, OFDM Simulator Using MATLAB , International Journal of Emerging Technology and Advanced Engineering, Volume 3, Issue 9, September 2013.

[17] Vibha Rao, T. Malavika, Performance analysis of MIMO-OFDM for multiple Antennas,International Journal of Advanced Research in Electrical, Electronics and Instrumentation Engineering, Vol. 3, Issue 5, May 2014

[18] Oomke Weikert and Udo Zolzer, A flexible laboratory MIMO system using four transmit four receive antennas, Proeedings of the 10th International OFDM-Workshop, August 2005, pp. 298-302 .

[19] Macro v.Barbera, Sokol Kosta, Alessandro Mei and Julinda Stefa, "To offload or Not to Offload? The Bandwidth and Energy Costs of Mobile Cloud Computing", Sapienza University of Rome, Italy.

[20] Abdul Nazir Khan, M.L Mat Kiah, Samee U. Khan and Sajjad A.Madani, "Towards secure mobile cloud computing: A survey", Elsevier Future Generation Computer Systems 29(2013) 1278-1299.

WIRELESS COMMUNICATIONS AND MOBILE COMPUTING

Wirel. Commun. Mob. Comput.

2013; 13:1587-161

\section{AUTHORS}

\section{Suremya Varghese}

She has obtained her Bachelors in Electronics and communication engineering in 1994 from Mahatma Gandhi University and currently doing her Masters in Digital electronics and advanced communication at, Manipal University Dubai Campus. She got 1 year teaching and 5 years industrial experience.

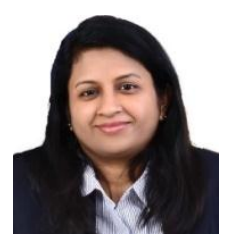

\section{Ganesan Subramanian:}

Prof. GANESAN .S obtained his Bachelors in Electronics and communication Engineering in 2000 from Madurai Kamaraj University, and Master of Engineering in Digital Communication \& Networking Engineering in 2004 from Anna University, Chennai. He has more than 15 years of teaching experience and published 20 research papers in International Journals. Currently he is working as Assistant Professor in the Engineering Department, Manipal University Dubai Campus. Area of Interest in Teaching: Cloud computing, Mobile Cloud, Information Theory and Coding Techniques, Wireless Communication, Wireless

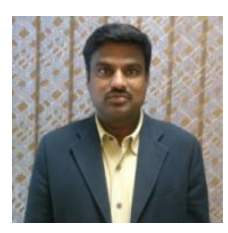
Sensor Networks 